\title{
Spatial Data Analysis and Modeling, Geoprocessing to Support Real Estate Decisions on Spatial Data of Tirana
}

\author{
Gerta Gradeci \\ Department of Informatics, \\ Faculty of Natural Sciences, \\ University of Tirana, Albania
}

\begin{abstract}
GIS has advanced as a new emerging field to the enhancement of information and communication technologies based on the spatial data implementations. Recently GIS is become indispensable field and is used in multidisciplinary areas to access information with relation to position. The rapid growth of the spatial data, information and communications has emerged to address spatial data analysis through GIS platforms, which are developing platforms for selection, integration, managing and analyzing massive amount of spatial data. In this paper, is presented on overview of the spatial data analysis and geoprocessing techniques used to provide tools and framework for performing analysis and managing geographic/spatial data. Also, is presented an approach and propose a spatial data modelling, which provides analysis capabilities to support real estate decisions based on spatial data of the capital city of Albania, Tirana.
\end{abstract}

\section{Keywords}

GIS, Spatial Data Analysis, Geoprocessing, Spatial Data Modeling, decision support

\section{INTRODUCTION}

The explosive growth of these spatial data and the widespread use of spatial databases emphasize the need for the automated spatial data analysis in many areas in different application domain. Geographical Information System (GIS) as developing platforms for selection, integration, managing and analyzing spatial data, contains heterogeneous data from multidisciplinary sources in different formats. A large amount of spatial data are being integrated and managed through GIS and this has been demanding to complex solutions and technologies, which perform spatial data analysis and geoprocessing tools.

The field of research consists in providing an overview of spatial data analysis and geoprocessing techniques in order to implement spatial data modelling and analysis. In this paper is proposed an approach, which aims and supports real estate decisions through the spatial data modelling.

The intent of this paper is also to provide an illustration of modelling the spatial data, implementation of spatial analysis and geoprocessing related to spatial data of capital city of Albania, Tirana.

\section{SPATIAL DATA ANALYSIS AND MODELING}

Geographic Information Systems (GIS) with new powerful technologies have been evolving and are quickly becoming part of a worldwide emergent digital infrastructure. Spatial data analysis is becoming more important than ever because enormous volumes of spatial data are available from different sources. [1] Geographical or spatial data play a vital role in many parts of daily life as we are dependent on information about where things are located and about the attributes of those things.

Spatial data analysis is a complex subject area and it can be difficult to separate from other fields because it takes elements from a range of academic disciplines, including geophysics, mathematics, astronomy, and cartography. When locational information is provided, known as spatial data, spatial analysis can be performed to calculate statistical and mathematical relationships through time and space. Methods of spatial analysis and modeling, integrated in a GIS environment, can be used to better understand reality and give rise and support decision making and planning in several domains. Making use of spatial data requires a whole set of approaches to extract information from those data and make them useful and underpinning these approaches is the analysis of data. [2]

Spatial analysis in GIS allows us to turn data into information and create new data as derivative datasets by manipulating existing spatial features and their related attributes. Spatial Data Analysis introduces key principles about spatial data, provides guidance on methods for their exploration, and provides a set of key ideas or frameworks that will give knowledge of the kinds of problems that can be tackled using the tools that are widely available for the analysis of spatial data. [2] GIS packages are equipped and offer a variety of analysis functions that allow us to manipulate both vector and raster data formats. These functions can be thought of as a set of tools for spatial analysis, and in fact several GIS applications use this "toolbox" analogy in describing the geoprocessing functions available. [3] It can be stated that spatial analysis is the process of geographically modeling a problem or issue, deriving results by technical spatial processing, and then examining and interpreting those model results. The spatial model that we create is based on a set of tools that apply operations on the spatial data to create new results. [4]

In a GIS framework, spatial modelling can provide insights about the way real systems work with enough precision and accuracy to permit prediction and assertive decision-making. [1] Modeling spatial problems can help us perform useful spatial analysis. In general terms, a model is a representation of reality. Models are created as a simplified and manageable view of reality due to the inherent complexity of the world and the interactions in it. Models help you understand, describe, and predict how things work in the real world. [4]

There are two main types of models:

- Representation models

Representation models try to describe the objects in a landscape. 
- Process models

Process models attempt to describe the interaction of the objects that are depicted in the representation model and the relationships are modeled using spatial analysis. Process models can be used to describe processes, but they are often used to predict what will happen if some action occurs.

Once is identified what type of model is needed to create and can solve the spatial problem, should then be identified the set of conceptual steps that can be used to help building that spatial model.

\section{SPATIAL DATA MODELLING AND GEOPROCESSING}

\subsection{A conceptual spatial data modelling as a methodological approach and geoprocessing on supporting real estate decisions}

The process of geographically modeling or spatial data modeling, which derives results by specific computer processing, then examining and interpreting these model results, is also defined as the process that performs spatial analysis. The spatial model created, is based in a set of tools that apply spatial data operations and create new results or derive new spatial data.

In this regard, as an essential part of ArcGIS platform is Geoproccesing. The fundamental purpose of geoprocessing is to provide tools and a framework for performing analysis and managing the geographic data. The modeling and analysis capabilities geoprocessing provides make ArcGIS a complete geographic information system. [9]

A typical geoprocessing tool performs an operation on a spatial dataset and produces new data as the result.

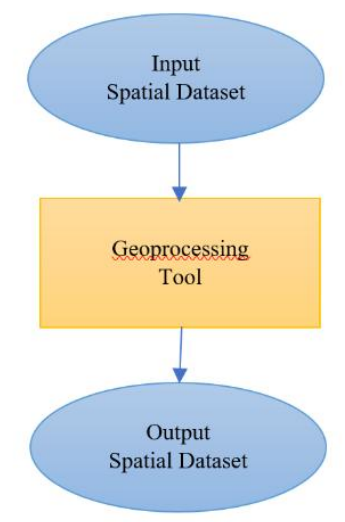

Fig 1: Geoprocessing tool

In this paper a conceptual spatial data modeling, as a methodological approach to solve specific spatial problem or supporting decisions. A set of conceptual steps is proposed, to be followed and used in order to help building the model.
- Stating the problem as sustainability analysis for acquiring a real estate object

Firstly is needed to state the spatial problem, that we are trying to solve and the goal we are trying to achieve.

- Criteria selection and screening

Once the problem is stated, should be performed setting the objectives for the selection and breaking down the spatial problem. So criteria selection and screening is defined on the spatial data.

- Exploring input datasets, setting the inputs as parameters Exploring input datasets involves understanding, which attributes within and between datasets are important for solving the problem and looking for trends in the data. Analysis should be performed, if setting the spatial input datasets as parameters is needed.

- Define process models on the spatial datasets For each objective set, the process model should be defined. The spatial datasets inputs can be defined also as parameters and this constructs a process. A model is built by connecting processes.

- Performing analysis, Geoprocessing

Once the objectives are decided, the elements and their interactions, the process models, and what input datasets will be needed, then at this point we are in the position to perform spatial analysis.

- Generating the result

When we have results from any spatial analysis, we should verify that it is correct. The final step in the spatial model is to implement the result, which consists in validating and generating the result.

In Fig. 2 is represented the conceptual flow chart, which aims the process for spatial data modeling.

\subsection{Case study: Modeling and geoproccessing on spatial data of Tirana}

Based on the above steps of the conceptual flow chart for spatial data modelling, a data model is developed using the ModelBuilder of ArcMap platform.

ModelBuilder is an application used to create, edit, and manage models. Models are workflows that string together sequences of geoprocessing tools, providing the output of one tool to another tool as input. ModelBuilder can also be thought of as a visual programming language for building workflows. [12]

This spatial data model, which it is developed, is a case study with the spatial data of the capital of Albania, Tirana municipality. This data model with its results is a tool, which the aim and its goal consists in supporting the decision making and the sustainability analysis for acquiring a real estate object.

In Fig. 3 is the map of Tirana with all the respective layers, which will be used as selection criteria and setting the input datasets for the model. 


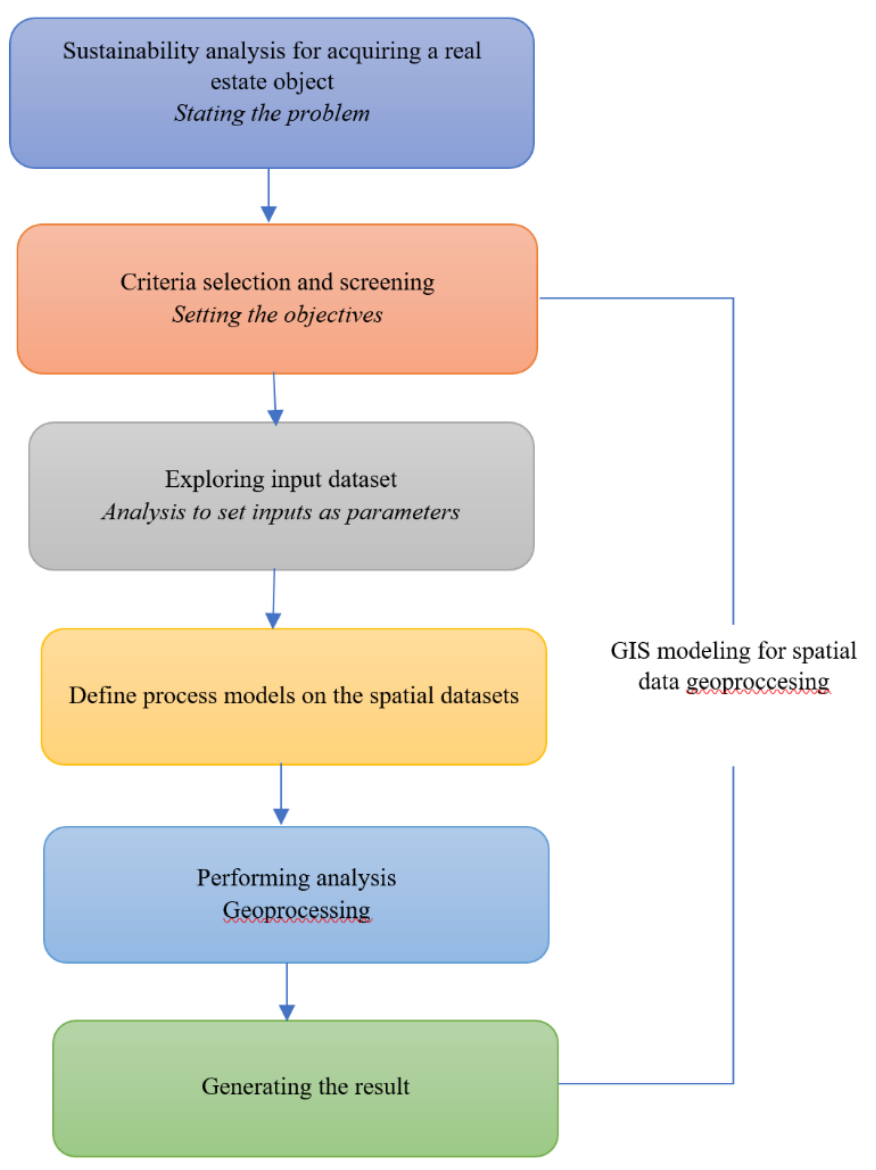

Fig 2: Conceptual flow chart for spatial data modeling

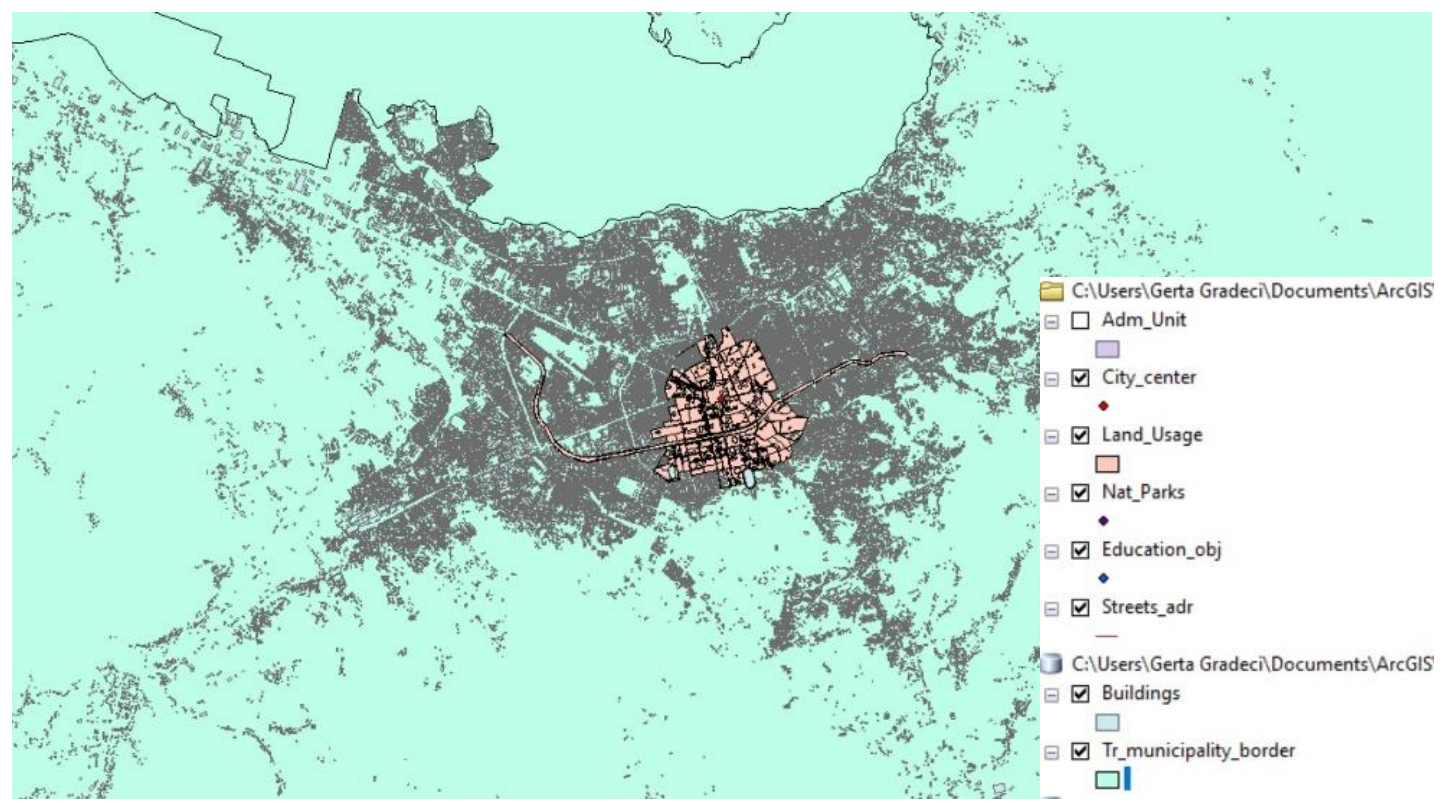

Fig 3: Map of Tirana with selected layers

In Fig. 4 is presented the spatial data model, developed using ModelBuilder of ArcMap platform and with main defined processes based on the analysis of the input datasets as the distance from city center, natural parks, education object, selection of intensity of construction. However, these spatial data inputs, which define the main model process and also the criteria of the geoprocessing tool such as the buffering and selection tools, are set as parameters. So, the tool implemented is customizable to support decisions on site selection of real estate objects. 


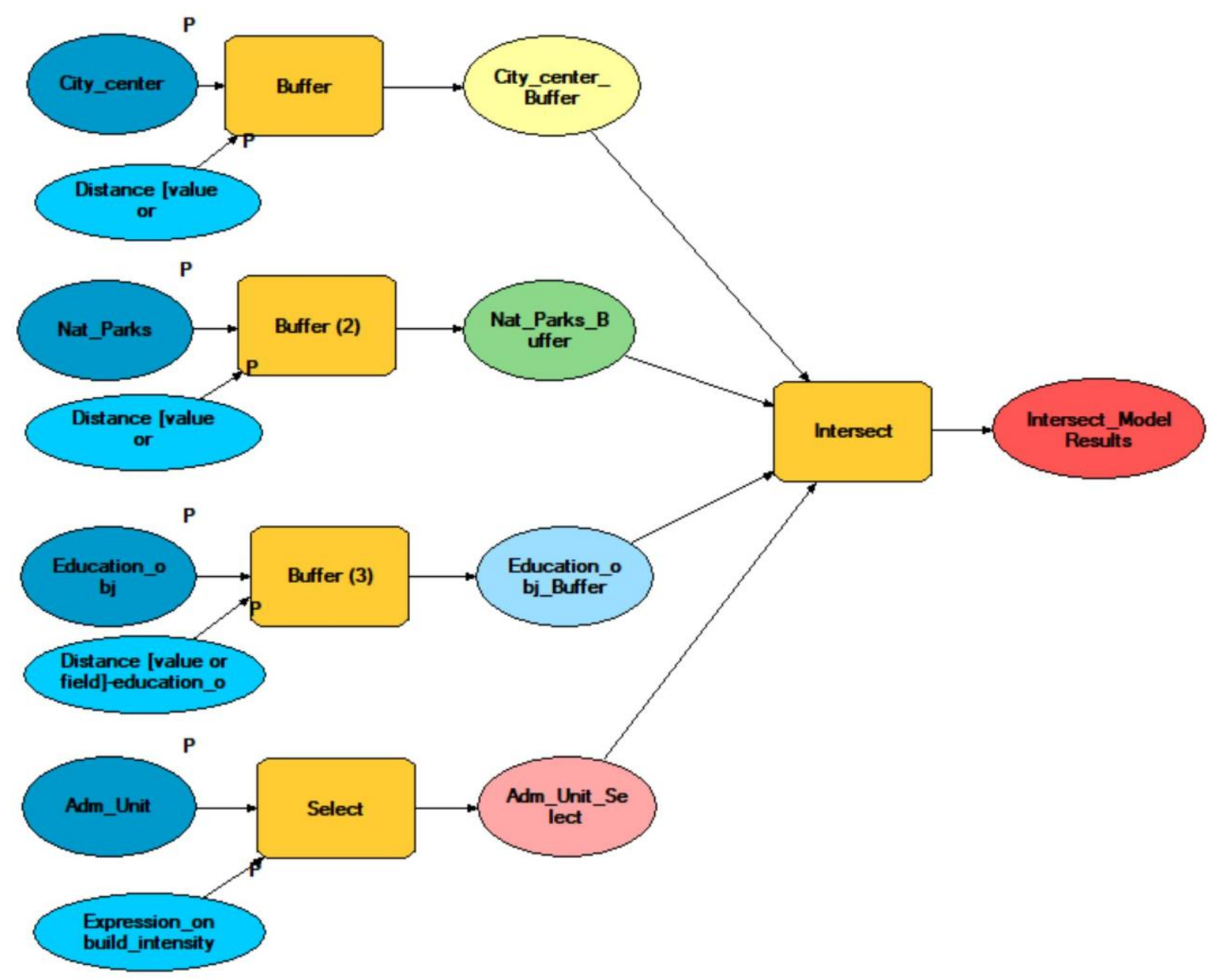

Fig 4: Spatial data model

In Fig. 5 is executed the buffer geoprocessing tool based on distance from city center, natural parks, education objects.

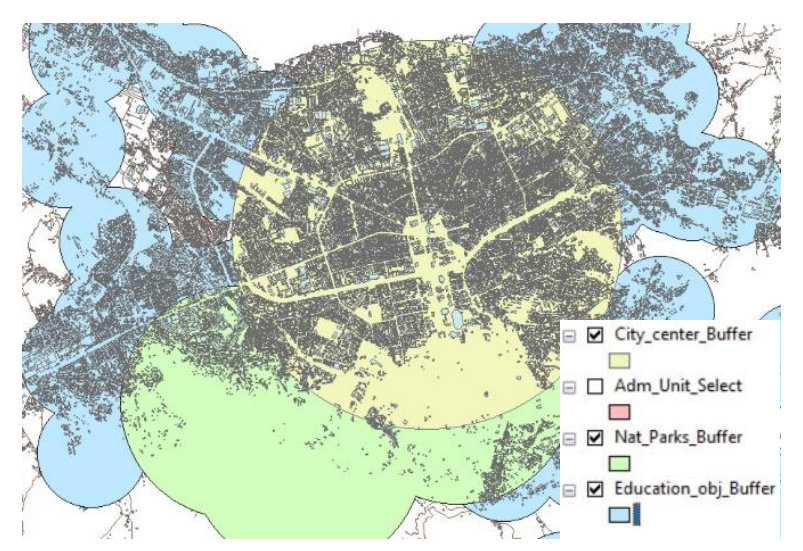

Fig. 5 Three model processes selected

In Fig. 6 is executed the select geoprocessing tool to view spatial data that meet the criteria of the construction intensity.

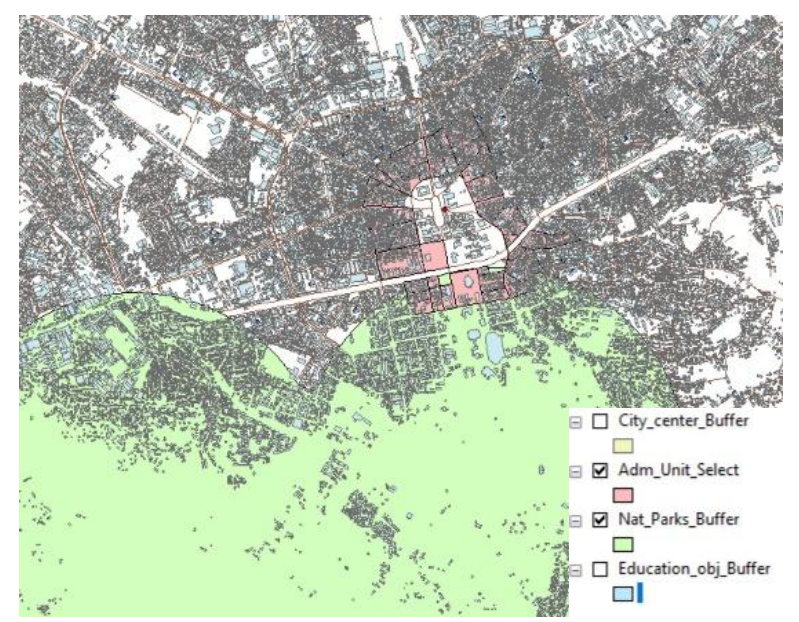

Fig. 6 Two model processes selected

In Fig. 7 are shown the results of the spatial data model developed as an intersection geoprocessing tool, which meet all criteria of the datasets input. 


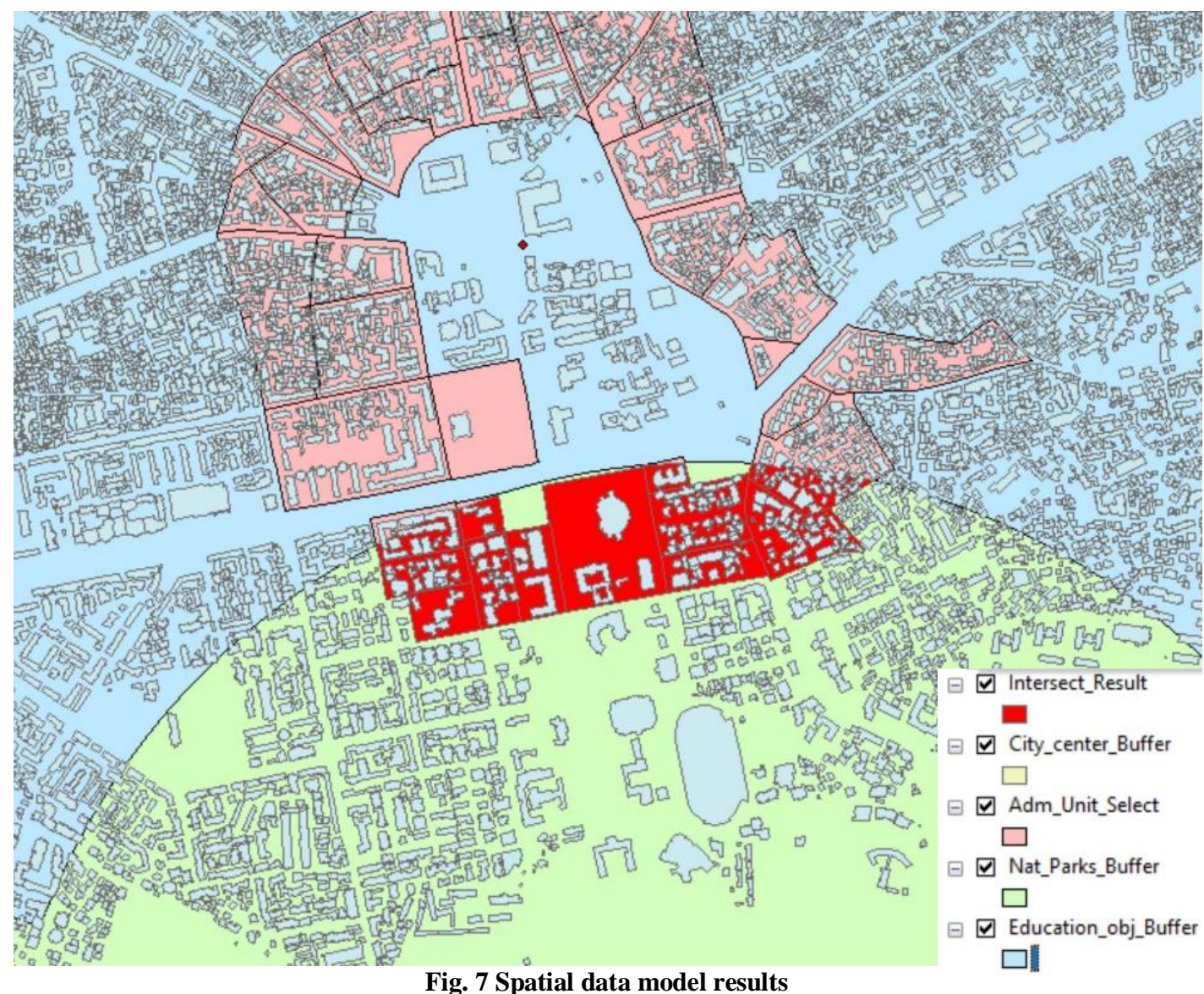

Below is shown the python script for executing the spatial data model:

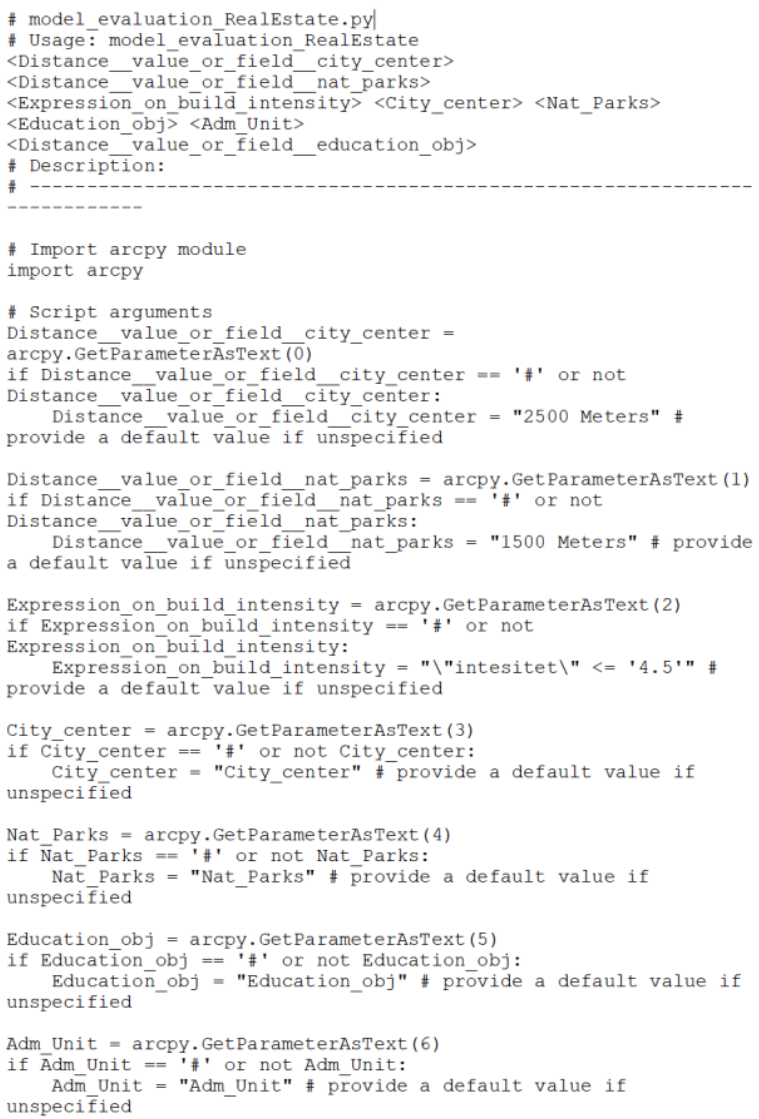

Distance_value_or_field_education_obj $=$

arcpy.GetParame $\overline{t e r} \overline{\mathrm{A}} \mathrm{sText} \overline{(7)}$

if Distance value or field education obj $==$ '\#' or not Distance_value_or_fieleld_education_obj Distance vālue or field education obj $=$ "700 Meters" \# provide a default value if unspecified

\# Local variables:

City center Buffer $=$ "C: $\backslash \backslash$ Users $\backslash \backslash$ Gerta Gradeci $\backslash \backslash$ Documents $\backslash \backslash$ ArcGIS

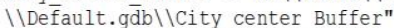

Nat_Parks_Buffer $=\overline{\text { C }}: \backslash \backslash$ Users $\backslash \backslash$ Gerta Gradeci $\backslash \backslash$ Documents $\backslash \backslash$ ArcGIS

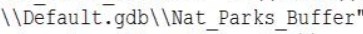

Education_obj_Buffer $=" \bar{C}: \backslash \backslash$ Users $\backslash \backslash$ Gerta Gradeci $\backslash \backslash$ Documents $\backslash \backslash$ ArCGIS $\backslash$ Default.gdb\\Education obj Buffer"

Adm Unit Select $=$ "C: $\backslash \backslash$ Users $\backslash \backslash$ Gerta Gradeci $\backslash \backslash$ Documents $\backslash \backslash$ ArcGIS $\backslash \backslash$ Default.gdb \\Adm Unit Select"

Intersect Model Results ${ }^{-}=$"C: $\backslash$ Users $\backslash \backslash$ Gerta Gradeci $\backslash$ Documents

$\backslash \backslash$ ArCGIS \} \backslash \text { Default.gdb\\Intersect Result" }

\# Process: Buffer

arcpy.Buffer analysis (City_center, City_center_Buffer, Distance_value_or_field_city_center, "FULL", "ROUND", "ALL", " ", "PLANAR")

\# Process: Buffer (2)

arcpy.Buffer analysis(Nat Parks, Nat Parks Buffer,

Distance value_or_field_nat_parks, "FULL", "ROUND", "ALL", "", "PLANAR")

\# Process: Buffer (3)

arcpy.Buffer_analysis(Education_obj, Education_obj_Buffer, Distance_value_or_field_education_obj, "FULL", "R̄ouND", "ALL", " ", "PLANAR" )

\# Process: Select arcpy.Select analysis(Adm Unit, Adm Unit Select, Expression on build intensity)

\# Process: Intersect

arcpy. Intersect analysis (" 'C: \\Users $\backslash \backslash$ Gerta Gradeci \Documents

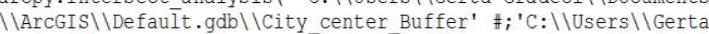

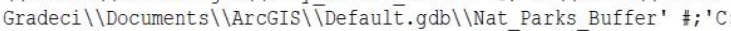
$\backslash \backslash$ Users $\backslash$ Gerta Gradeci \\Documents \\ArcGIS \Default.gdb \\Education obj Buffer' \#; 'C: \Users \Gerta Gradeci\\Documents $\backslash \backslash$ ArcGIS $\backslash \backslash$ Default.gdb $\backslash \backslash$ Adm Unit Select' \#",

Intersect Model Results, "ĀLL", "n", "INPUT") 


\section{CONCLUSIONS}

In this paper is discussed related to the spatial data analysis as a complex subject area and the importance of this field, as allow to turn spatial data into information based on creating new derivative datasets by manipulating existing spatial features and their related attributes. Modeling the spatial problems is helpful to perform spatial analysis. Spatial analysis and modeling, integrated in a GIS environment, is used to better understand reality and give rise and support decision making and planning in several domains.

In addition, is presented a conceptual spatial data modeling with proposed steps to follow, as a methodological approach to solve specific spatial problem or supporting decisions. Based on this, is developed a spatial data model performing analysis and geoprocessing as a case study with the spatial data of the capital of Albania, Tirana. This spatial data model with its derivative results from geoproccesing aims supporting decision making and the sustainability analysis for acquiring a real estate object.

Future work will consist in further enhancement of spatial analysis and geoprocessing techniques based on spatial databases.

\section{REFERENCES}

[1] Jorge Rocha and José António Tenedório, 2018. Spatial Analysis, Modelling and Planning

[2] Christopher Lloyd, 2010, Spatial Data Analysis: An Introduction for GIS users

[3] Ann Blyth, Dave Cake, Ian Laing, Martin Andresen, Gennady Gienko, Michael Govorov. 2008. Spatial Analysis And Modeling - training material

[4] Solving spatial problems with representation and process models,

https://desktop.arcgis.com/en/arcmap/latest/extensions/sp atial-analyst/solving-problems/using-the-conceptualmodel-to-create-suitability.htm, www.esri.com

[5] Penjani Hopkins Nyimbili, Turan Erden. 2020. A combined model of GIS and fuzzy logic evaluation for locating emergency facilities: a case study of Istanbul

[6] Martin Behnisch, Gotthard Meinel. 2018. Trends in Spatial Analysis and Modelling

[7] Elzbieta Bielecka, 2020, GIS Spatial Analysis Modeling for Land Use Change. A Bibliometric Analysis of the Intellectual Base and Trends

[8] Hemlata Goyal, Chilka Sharma, Nisheeth Joshi. 2017. An Integrated Approach of GIS and Spatial Data Mining in Big Data

[9] What is geoprocessing? Esri 2020 , https://desktop.arcgis.com/en/arcmap/latest/analyze/main /what-is-geoprocessing.htm

[10] Esri 2020. Solving spatial problems with representation and process models https://desktop.arcgis.com/en/arcmap/latest/extensions/sp atial-analyst/solving-problems/solving-spatial-problemswith-representation-and-process-models.htm

[11] Esri 2020, Using the conceptual model to create a suitability map. https://desktop.arcgis.com/en/arcmap/latest/extensions/sp atial-analyst/solving-problems/using-the-conceptualmodel-to-create-suitability.htm

[12] Esri 2020. What is ModelBuilder? https://desktop.arcgis.com/en/arcmap/latest/analyze/mod elbuilder/what-is-modelbuilder.htm

[13] Esri 2020. Creating tools with ModelBuilder. https://desktop.arcgis.com/en/arcmap/latest/analyze/creat ing-tools/creating-tool-with-modelbuilder-tutorial.htm

[14] Esri 2020. Executing tools in ModelBuilder. https://desktop.arcgis.com/en/arcmap/latest/analyze/exec uting-tools/executing-tools-in-modelbuilder-tutorial.htm

[15] Eric Pimpler, 2013, Programming ArcGIS 10.1 with Python Cookbook 